Pacific Journal of Mathematics

SOME RESULTS ON BLOCKS OVER LOCAL FIELDS 


\title{
SOME RESULTS ON BLOCKS OVER LOCAL FIELDS
}

\author{
W. M. HubBarT
}

\begin{abstract}
Let $F$ be an unequal characteristic local field. The aim of this paper is to outline a block form of the Cartan-Brauer modular decomposition theory which incorporates the notion of defect groups. The irreducible $F$-representations of a finite group $G$ are associated with blocks in the group algebra over the residue field $\bar{F}$. The defect groups of a block to which an irreducible $F$-representation $T$ belongs are shown to coincide with the defect groups of the block to which any absolutely irreducible constituent of $T$ belongs. A result on the Schur index of an absolutely irreducible representation belonging to a block of defect zero is proven which yields an analogue to the Brauer-Nesbitt Theorem on blocks of defect zero. The number of $\bar{F}$-blocks of highest defect is shown to be equal to the number of $p$-regular $\bar{F}$-conjugacy classes of highest defect.
\end{abstract}

Let $G$ be a finite group and let $F$ be an unequal characteristic local field; i.e., a field of characteristic zero, complete with respect to a discrete non-Archimedean valuation, whose residue field $\bar{F}$ is of characteristic $p$. The aim of this paper is to initiate a study of a block form of the Cartan-Brauer decomposition theory $[2, \S 83 \mathrm{~A}]$ which incorporates the notion of defect groups. Defect groups for $\bar{F}$-blocks are defined as in [9]. The irreducible $F$-representations and the indecomposable $\bar{F}$-representations are associated with $\bar{F}$-blocks. In Theorem 2 we show that the defect groups of a block to which an irreducible $F$-representation $T$ belongs are the same as the defect groups of the blocks to which the absolutely irreducible constituents of $T$ belong. Using Theorem 2 and assuming that the residue field $\bar{F}$ is perfect, we obtain a result on the Schur index which yields an analogue to the Brauer-Nesbitt Theorem on blocks of defect zero. [2, 86.3]. In $\S 4$ we use some lifting techniques together with a permutation lemma of Brauer to show that the number of $\bar{F}$-blocks of highest defect is equal to the number of $p$ regular $\bar{F}$-conjugacy classes of highest defect. This last result has been obtained independently by W. F. Reynolds as a consequence of some work on blocks in twisted group algebras [8]. Also L. G. Kovacs in some unpublished notes has obtained the result for blocks in group algebras over finite fields using the theory of vertices and sources.

Some of the results in this paper are essentially contained in a thesis presented to the Department of Mathematics and the Graduate School of the University of Oregon in partial fulfillment of the requirements for the degree of Doctor of Philosophy. The author wishes to take this opportunity to thank Professor C. W. Curtis for his valu- 
able assistance and kind encouragement.

First we make some preliminary definitions and observations. Denote the conjugacy classes of $G$ by $\mathscr{C}_{1}, \cdots, \mathscr{C}_{t}$ and let $C_{i}$ be the class sum corresponding to the class $\mathscr{C}_{i}$. Let $F$ be a field of characteristic $p$. Primitive idempotents in the center $Z(F G)$ of the group algebra $F G$ are called $F$-block idempotents, or more simply, $F$-blocks. Suppose $E$ is a Galois extension of $F$. Denote the Galois group of $E$ over $F$ by $\mathscr{G}(E / F)$. Each $\sigma \in \mathscr{C}(E / F)$ induces an automorphism of the group algebra $E G$ which permutes the $E$-block idempotents. The following result characterizes $F$-blocks with a given defect group in terms of $E$-blocks.

Proposition 1. [7, Theorem 2] Let $\mathscr{D}$ be the set of E-blocks whose defect groups are conjugate to some p-subgroup $D$ of $G$. Then $\mathscr{G}(E / F)$ acts as a permutation group on $\mathscr{D}$ and the orbits of $\mathscr{D}$ relative to $\mathscr{C}(E / F)$ are in one-to-one correspondence with the set of $E$ blocks whose defect groups are conjugate to $D$. If $\mathcal{O}$ is an orbit, then $e=\Sigma d$, where the sum is over $\mathcal{O}$, is the $F$-block corresponding to 0 .

Proof. The fact that $F$-blocks correspond to orbits of $E$-blocks relative to the action of $\mathscr{C}(E / F)$ is a consequence of a well-known result about idempotents in commutative algebras [10, § 121]. If an $E$-block $d$ is written as in [9,3.2], it is easy to see that $d$ and $\sigma(d)$ have the same defect groups, and hence that each $\sigma \in \mathscr{G}(E / F)$ parmutes

Let $\Omega$ be an algebraic closure of $E$. If $e$ is an $F$-block, then $Z(F G) e$ is a completely primary algebra and so $Z(F G) e / \operatorname{rad} Z(F G) e$ is a finite extension of $F$. The natural homomorphism $\psi$ of $Z(F G)$ onto $Z(F G) e / \operatorname{rad} Z(F G) e$ has as its kernel the maximal ideal $Z(F G)(1-e)+$ $\operatorname{rad} Z(F G)$. Any homomorphism from $Z(F G)$ into $\Omega$ with kernel $Z(F G)(1-e)+\operatorname{rad} Z(F G)$ is said to be associated with $e$. Observe that if $\psi$ is associated with $e$, then $\psi(e)=1$.

Let $e=\Sigma \sigma_{i}(d)$, where the sum is over an orbit $\mathcal{O}$, be an $F$-block. Write

$$
e=\sum_{i \in A} f_{i} C_{i}+\sum_{j \in B} f_{j} C_{j}
$$

and

$$
d=\sum_{i \in A^{\prime}} b_{i} C_{i}+\sum_{j \in B^{\prime}} b_{j} C_{\jmath}
$$

as in $[9,3.2]$. To show that $e$ and $d$ have defect groups conjugate 
to $D$ it suffices to show that $A \cap A^{\prime} \neq \varnothing$. We have $f_{i}=\Sigma b_{i}{ }^{\sigma_{j}}$. Thus if $f_{i} \neq 0$, then $b_{i} \neq 0$, and so $A \cup B \subseteq A^{\prime} \cup B^{\prime}$. Therefore, $A \subseteq A^{\prime} \cup B^{\prime}$.

Now assume $A \subseteq B^{\prime}$. Let $\eta$ be a homomorphism from $Z(E G)$ into $\Omega$ associated with $d$. Since $A \subseteq B^{\prime}$, we have by $[9,3.2]$

$$
\eta(e)=\sum_{i \in A} f_{i} \eta\left(C_{i}\right)+\sum_{j \in B} f_{j} \eta\left(C_{j}\right)=0 .
$$

But the restriction of $\eta$ to $Z(F G)$ is a homomorphism associated with $e$, a contradiction. Thus $A \subsetneq B^{\prime}$ and $A \cap A^{\prime} \neq \varnothing$ and the proof is complete.

2. Block idempotents and representations. Let $F$ be a local field of unequal characteristic. Denote the ring of integers in $F$ by $O_{F}$ the unique maximal ideal by $P_{F}$ and the residue field $O_{F} / P_{F}$ by $\bar{F}$. The natural map $\alpha \rightarrow \bar{\alpha}$ from $O_{F}$ onto $\bar{F}$ induces a homomorphism from the group algebra $O_{F} G$ onto $\bar{F} G$. By [2, $\left.\S 77\right]$ there is a one-toone correspondence between $O_{F}$-blocks and $\bar{F}$-blocks, the correspondence being given by $e \leftrightarrow \bar{e}$. Here $O_{F}$-blocks are primitive central idempotents in the group algebra $O_{F} G$.

An irreducible $F G$-module $X$ is isomorphic to a minimal left ideal in some simple component $A$ of $F G$. The identity $\varepsilon$ of $A$ is a primitive central idempotent in $F G$. The module $X$ is said to be associated with $\varepsilon$. Observe thet an $O_{F}$-block $e$ is a central idempotent in $F G$, and hence $e$ is the sum of a uniquely determined set of primitive central idempotents in $F G$. An irreducible $F G$-module $X$ belongs to an $\bar{F}$-block $\bar{e}$ provided the primitive central idempotent $\varepsilon$, with which $X$ is associated, is a summand of $e$. If $X$ affords a character $\chi$, we shall also say that $\chi$ belongs to $\bar{e}$. An indecomposable $\bar{F} G$-module $M$ belongs to $\bar{e}$ in case $\bar{e} M \neq 0$.

Let $X$ be an indecomposable $O_{F} G$-submodule of an irreducible $F G$ module $X^{*}$ obtained by $[2,73.6]$. As in $[2, \S 85]$ we form the $\bar{F} G$ module $\bar{X}$; it can be shown that the $\bar{F}$-block to which the $\bar{F} G$-composition factors of $\bar{X}$ belong is the same as the $\bar{F}$-block to which $X^{*}$ belongs. Thus, we can establish a block form of the decomposition theory of $[2, \S 83 \mathrm{~A}]$. The hypothesis that $F$ is complete is necessary for such a block decomposition theory; for if $T$ is the irreducible $Q$ representation of a cyclic group of order $p-1$ associated with the cyclotomic polynomial $\Phi_{p-1}(x)$, then the composition factors of $\bar{T}$ lie in different $\bar{Q}=Z_{p}$ blocks.

We return to the case where $F$ is complete. All algebraic extensions of $F$ will be assumed to lie in a fixed algebraic closure $\Omega$ of $F$. Denote the unique extension of the valuation on $F$ to $\Omega$ by $\phi$, and for an extension $L$ set 


$$
\begin{gathered}
O_{L}=\{a \in L \mid \dot{\phi}(a) \leqq 1\} \\
P_{L}=\{a \in L \mid \dot{\phi}(a)<1\} \\
\bar{L}=O_{L} / P_{L} .
\end{gathered}
$$

For $\alpha \in O_{L}$ set $\bar{\alpha}=\alpha+P_{L}$. We fix the following notation for the remainder of the paper. Let $\omega$ be a primitive $n$th root of unity where $n=p^{a} m$ with $(p, m)=1$ is the exponent of $G$. Set $E=F(\omega)$ and $K=F\left(\omega^{p^{a}}\right)$. From the extension theory of complete fields [11, Chapter 2] it follows that elements $\sigma$ of $\mathscr{Y}(K / F)$ map $O_{K}$ onto $O_{\sigma K}=O_{K}$ and $P_{K}$ onto $P_{\sigma K}=P_{K}$. The map $\bar{\sigma}$ defined by $\bar{\sigma}(\bar{\alpha})=\overline{\sigma(\alpha)}$ is an automorphism of $\bar{K}$. Since $\bar{K}$ is separable over $\bar{F}$, the map $\sigma \rightarrow \bar{\sigma}$ is a homomorphism from $(K / F)$ onto $(\bar{K} / \bar{F})$, [11,3-5-6]. The fact that $K$ is unramified over $F$ implies that $\mathscr{C}(K / F)$ and $(\bar{K} / \bar{F})$ are isomorphic. Also since $E$ is totally ramified over $K$, it follows that $\bar{E}=\bar{K}$; thus, the $\bar{E}$-blocks are the same as the $\bar{K}$-blocks. Since $E$ is a splitting field for $G$, the absolutely irreducible representations of $G$ belong to $\bar{E}$-blocks, which by the preceding observation are actually $\bar{K}$-blocks.

THEOREM 2. Let $\chi$ be a irredusible F-character with an absolutely irreducible constituent $\zeta$. Then the defect groups of the $\bar{F}$-block to which $\chi$ belongs are the same as the defect groups of the $\bar{K}$-block to which $\zeta$ belongs.

Proof. Since $E$ is a splitting field for $G$, $\zeta$ is afforded by an irreducible $E G$-module. By [3, Theorem 1.4] $\chi=m_{F}(\zeta) \Sigma \zeta^{\sigma_{i}}$ where the sum is over a full set of conjugates of $\zeta$ by $(E / F)$ and $m_{F}(\zeta)$ is the schur index of $\zeta$ relative to $F$.

If $\partial$ and $\varepsilon$ are the primitive central idempotents in $E G$ and $F G$ with which $\zeta$ and $\chi$ are associated respectively, then $\varepsilon=\Sigma \sigma_{i}(\delta)$.

New let $\bar{e}$ and $\bar{d}$ be the $\bar{F}$ and $\bar{K}$-blocks to which $\chi$ and $\zeta$ belong respectively. Since $\delta$ is an $E$-summand of $\varepsilon$, and $\varepsilon$ is an $F$-summand of the $O_{F}$-block $e$; it follows that $\delta$ is an $E$-summand of $e$. If $e=\Sigma d_{j}$ is a decomposition of $e$ into $O_{K}$-blocks, then one easily verifies that $j$ is an $E$-summand of some $d_{j}$. Thus $d=d_{j}$ and by Proposition $1, \bar{e}$ and $\bar{d}$ have the same defect groups.

In the following two sections we shall say that a block $\bar{e}$ has defect $a$ in cass each of its defect groups have order $p^{a}$.

3. Defect zero. Throughout this section we assume that the residue field $\bar{F}$ is perfect. Since $\bar{K}$ is the residue field of $E$ and $E$ is a splitting field for $G$, it follows that the Brauer-Nesbitt Theorem [2, 86.3] holds for $\bar{K}$-blocks. Let $\bar{e}$ ba an $\bar{F}$-block of defect zero. By Proposition 1, we have $\bar{e}=\sum_{r=1}^{r} \bar{\sigma}_{\imath}(\bar{d})$ where the $\bar{\sigma}_{\imath}^{\prime}$ s are chosen so that $\left\{\bar{\sigma}_{i}(\bar{d})\right\}_{\imath=:}^{r}$ is an orbit of $\bar{K}$-blocks each of defect zero. 
Lemma 3. The $\bar{F}$-block $\bar{e}$ contains exactly one irreducible $F G$ module, one irredusible $\bar{F} G$-module, and one projective indecomposable module. If $M$ is the unique irreducible $\bar{F} G$-module belonging to $\bar{e}$, then

$$
M^{\bar{K}} \cong \sum_{i=1}^{r} V^{\bar{\sigma}_{i}}
$$

where $V$ is the unique irredusible $\bar{K} G$-module belonging to $\bar{d}$ and the $\bar{\sigma}_{i}^{\prime}$ s are determined by the $\bar{K}$-decomposition of $\bar{e}$.

Proof. Observe that $\bar{e}=\sum_{i=1}^{r} \bar{\sigma}_{i}(\bar{d})$ where $\left\{\sigma_{i}(d)\right\}_{i=1}^{r}$ is an orbit of $O_{K}$-blocks relative to $(K / F)$. Since $\bar{d}$ contains only one irreducible $E G$-module [2, 86.3], it follows that $d$ is itself a primitive central idempotent in both $E G$ and $K G$. Therefore, $e$ is a primitive central idempotent in $F G$; hence, $\bar{e}$ contains only the irreducible $F G$-module associated with the primitive central idempotent $e$.

Let $V$ be the unique irreducible $\bar{K} G$-module which belongs to $\bar{d}$ [2, 86.3]. For $\bar{\sigma} \in \mathcal{C}(\bar{K} / \bar{F})$, let $V^{\bar{\sigma}}$ ba a $\bar{K} G$-module affording the representation $T^{\bar{\sigma}}$ where $T$ is a representation afforded by $V$. Here $T^{\bar{\sigma}}(g)$ is the matrix obtained by conjugating the entries of $T(g)$ by $\bar{\sigma}$. Now each $V^{\bar{\sigma}_{i}}$ belongs to $\bar{\sigma}_{i}(\bar{d})$, and by the fact that each $\bar{\sigma}_{i}(\bar{d})$ has defect zero and hence $[2,83.3]$ contains only $V^{\bar{\sigma}_{i}}$, it follows that $\left\{V^{\left.\bar{\sigma}_{i}\right\}_{i=1}^{r}}\right.$ is a full set of distinct conjugates of $V$ by $(\bar{K} / \bar{F})$.

By [3, Theorem 1.4] and the fact that the Schur index $m_{\bar{F}}(V)=1$, it follows that

$$
M^{\bar{K}} \cong \sum_{i=1} V^{\bar{\sigma}}{ }_{i}
$$

for some irreducible $\bar{F} G$-module $M$. Since the $V^{\bar{\sigma}_{i}}$ are uniquely determined up to isomorphism by the $\bar{K}$-blocks $\bar{\sigma}_{i}(\bar{d})$, it follows by [2, 29.11] that $M$ is the unique irreducible $\bar{F} G$-module belonging to $\bar{e}$. The fact that $\bar{e}$ contains only one projective indecomposable $\bar{F} G$-module follows from $[2, \S 55]$.

Lemma 4. Let $L$ be an arbitrary field and let $N$ be an arbitrary irreducible LG-module having an absolutely irredusible constituent $W$ which affords a character $\omega$. Then $\left(\operatorname{Hom}_{L G}(N, N): L\right)=m_{L}^{2}(W)(L(\omega): L)$, where $m_{L}(W)$ is the Schur index of $W$ relative to $L$.

Proof. For characteristic zero see [2, $\S 70$ especially p. 469]. For characteristic $p$, let $C$ ba a Galois extension of $L$ so that $C$ is a splitting field for $G$ and suppose that $W$ is an irreducible $C G$-module. Then by [3, Theorem 1.4] $N^{C} \cong \Sigma W^{\sigma_{i}}$ where the sum is over a full set of conjugates of $W$ by $(C / L)$. 


$$
\operatorname{Hom}_{L G}(N, N) \otimes_{L} C \cong \operatorname{Hom}_{C G}\left(N^{c}, N^{c}\right)
$$

by $[2,29.5]$. Since $W^{\sigma_{i}} \neq W^{\sigma_{j}}$ for $i \neq j,\left(\operatorname{Hom}_{C^{\prime(i}}\left(N^{c}, N^{c}\right): C\right)$ is equal to the number of distinct conjugates of $W$. By [2, 30.15] this is the number of distinct conjugates of $W$ by $(C / L)$. But from Galois theory this is $(L(\omega): L)$. Thus $\left(\operatorname{Hom}_{L G}(N, N): L\right)=(L(\omega): L)$ and the lemma is proven.

Let $X^{*}$ be the unique irreducible $F G$-module belonging to $\bar{e}_{\text {. }}$ Choose an indecomposable $O_{F} G$-submodule $X$ with $X^{*}=F X$ and $\left(X: O_{F}\right)=\left(X^{*}: F\right)[2,73.6]$. Denote by $M$ and $U$ respectively the unique irreducible and projective indecomposable $\bar{F} G$-modules belonging to $\bar{e}$.

THEOREM 5. Let $\zeta$ be an absolutely irreducible character belonging to a block of defect zero. Then $m_{F}(\zeta)=1$.

Proof. Let $\chi$ be the irreducible $F$-character of which $\zeta$ is a constituent. By [3, Theorem 1.4] $\chi=m_{\Gamma}(\zeta) \sum_{i=1}^{r} \zeta^{\sigma_{2}}$ where $\left\{\zeta^{\sigma_{2}}\right\}_{2=1}^{\prime}$ is a full set of conjugates of $\zeta$ by $(E / F)$. Since $\zeta$ belongs to a block of defect zero, we have by $[2,83.6] \zeta(g)=0$ for non $p$-regular $g$. Since $\zeta(g) \in K$ for $p$-regular $g$, we may assume that each $\sigma_{i} \in \mathscr{C}(K / F)$. Let $\bar{d}$ be the $\bar{K}$-block to which $\zeta$ belongs. Observe that the restriction of $\zeta$ to the p-regular element of $G$ is the Brauer character of the unique irreducible $\bar{K} G$-module $V$ which belongs to $\bar{d}$. [2, 83.6] Also since each $\bar{\sigma}_{i}(\bar{d})$ has defect zero, $\zeta$ is the Brauer character of $V^{\bar{\sigma}}$. It follows by Lemma 3 that $\mu=\Sigma \zeta^{\rho_{i}}$ is the Brauer character of the unique irreducible $F G$-module $M$ which belongs to $\bar{e}$. Since $\chi=m_{F}(\zeta) \mu$, it follows that the multiplicity of $M$ in $\bar{X}$ is $m_{F}(\zeta)$.

Since $X^{*}$ belongs to a block of defect zero, the $O_{F} G$-module $X$ is projective [5, Corollary to 4.1a]. Select a primitive idempotent $\varepsilon \in O_{F} G$ so that $X \cong O_{F} G \varepsilon$. Since $X^{*}=F X$, it follows that $X^{*} \cong F G \varepsilon$, and so by $[2,54.19]$ we have

$$
\left(\varepsilon X^{*}: F\right)=\left(\operatorname{Hom}_{F G}\left(X^{*}, X^{*}\right): F\right) .
$$

Also by $[2, \S 77] \bar{\varepsilon}$ is primitive idempotent in $\bar{F} G$ and hence $U \cong \bar{F} G \bar{\varepsilon}$. Using the fact that $\bar{F}$ is perfect, we apply $[2,54.19]$ again to conclude that

$$
(\bar{\varepsilon} \bar{X}: \bar{F})=d \cdot\left(\operatorname{Hom}_{\bar{F} G}(M, M): \bar{F}\right)
$$

where $d=m_{F}(\zeta)$ is the multiplicity of $M$ in $\bar{X}$. Now by Lemma 4, $\left(\operatorname{Hom}_{F^{\prime} G}(X, X): F^{\prime}\right)=m_{F}^{2}(\zeta)(F(\zeta): F)$ and $\left(\operatorname{Hom}_{\bar{F} G}(M, M): \bar{F}\right)=(\bar{F}(\bar{\zeta}): \bar{F})$. Since $\left(\varepsilon X^{*}: F\right)=(\bar{\varepsilon} \bar{X}: \bar{F})$ and $(F(\zeta): F)=(\bar{F}(\bar{\zeta}): \bar{F})$, we have by $(3.1)$, and (3.2), 


$$
m_{F}^{2}(\zeta)=m_{F}(\zeta)
$$

Thus, $m_{F}(\zeta)=1$ and Theorem 5 is proven.

Corollary. Let $G$ be a finite group with a unique $\bar{K}$-block $\bar{d}$ of defect zero, and let $\zeta$ be the absolutely irreducible character belonging to $\bar{d}$. Then $\zeta$ is a rational-valued and the representation affording $\zeta$ is realizable in the p-adic completion $Q_{p}$ of the rational field.

Theorem 6. Let $\bar{e}$ be an $\bar{F}$-block of defect zero. Then $\bar{e}$ contains exactly one irreducible $F G$-module $X^{*}$, one irreducible $\bar{F} G$-module $M$, and one projective indecomposable module $U$. Moreover, $\bar{X} \cong U \cong M$.

Proof. The first part is Lemma 3. That $\bar{X} \cong U \cong M$ follows from the fact that $m_{F}(\zeta)=1$. For then Brauer character $\mu$ of $M$ is equal to the Brauer character $\chi$ of $\bar{X}$.

4. Highest defect. Our notation is as in $\S 2$. Observe that the Galois group $\mathscr{G}(\bar{K} / \bar{F})$ is isomorphic to a subgroup of $U_{m}$, the units modulo $m$. In $\S 4$ we let $\sigma_{i}$ denote the element of $\mathscr{C}(\bar{K} / \bar{F})$ which maps a primitive $m^{\text {th }}$ root of one to its $i^{\text {th }}$ power. Two $p$-regular elements $x$ and $y$ of $G$ are said to be $\bar{F}$-conjugate in case $x^{i}=g y g^{-1}$ for some $g \in G$ and $\sigma_{i} \in \mathscr{G}(\bar{K} / \bar{F}) . \quad \bar{F}$-conjugacy is an equivalence relation on the p-regular elements of $G$ and the equivalence classes are called p-regular $\bar{F}$-conjugacy classes. A defect group of an $F$-class $\mathscr{K}$ is any $p$-Sylow subgroup of the centralizer $C(x)$ where $x \in \mathscr{K}$. We estabilsh the following result using an argument similar to that in [4, 12.3].

THeOREM 7. The number of $\bar{F}$-blocks of highest defect is equal to the number of p-regular $\bar{F}$-conjugacy classes of highest defect.

Proof. By [9, Theorem 6.1] the number of $\bar{K}$-blocks of highest defect is equal to the number of $p$-regular conjugacy classes of highest defect. Let $\left\{\mathscr{C}_{1}, \cdots, \mathscr{C}_{t}\right\}$ be the ordinary $p$-regular conjugacy classes of highest defect and let $\left\{\bar{d}_{1}, \cdots, \bar{d}_{t}\right\}$ be the $\bar{K}$-blocks of highest defect. Now write each $\bar{d}_{i}$ as in $[9,3.2]$.

$$
\bar{d}_{i}=\sum_{j=1}^{t} b_{i}\left(g_{j}\right) C_{j}+\sum_{k \in B} b_{k} C_{k}, \quad \quad g_{j} \in \mathscr{C}_{j} .
$$

Let $B$ be the $t \times t$ matrix

$$
B=\left(b_{i}\left(g_{j}\right)\right) .
$$

Using the homomorphisms associated with the $\bar{d}_{i}^{\prime} s$ in the proof of Pro- 
position 1 , one can show that the rows of $B$ are independent, and thus that $B$ is nonsingular.

By Proposition 1, $\mathscr{C}(\bar{K} / \bar{F})$ acts as a permutation group on the set $\left\{\bar{d}_{i}\right\}$ and the orbits of $\left\{\bar{d}_{i}\right\}$ relative to $\mathscr{C}(\bar{K} / \bar{F})$ are in one-to-one correspondence with the $\bar{F}$-blocks of highest defect. We observe that the action of $\mathscr{C}(\bar{K} / \bar{F})$ on $\left\{\bar{d}_{i}\right\}$ induces an action of $\mathscr{G}(\bar{K} / \bar{F})$ on the rows of $B$, and thus the orbits of the rows of $B$ relative to $\mathscr{C}(\bar{K} / \bar{F})$ are in one-to-one correspondence with the $\bar{F}$-blocks of highest defect.

For a $p$-regular class $\mathscr{C}_{j}$ and $\sigma_{i} \in \mathscr{C}(\bar{K} / \bar{F})$ define $\sigma_{i}\left(\mathscr{C}_{j}\right)=\mathscr{C}_{k}$ where $x^{i} \in \mathscr{C}_{k}$ for $x \in \mathscr{C}_{j}$. We observe that the action of $\mathscr{C}(\bar{K} / \bar{F})$ on the $p$ regular classes preserves defect groups. The orbits of the set $\left\{\mathscr{C}_{i}\right\}_{i=1}^{t}$ relative to $\mathscr{C}(\bar{K} / \bar{F})$ are in one-to-one correspondence with $p$-regular $\bar{F}$-conjugacy classes of highest defect. Also the action of $\mathscr{G}(\bar{K} / \bar{F})$ on $\left\{\mathscr{C}_{i}\right\}_{i=1}^{t}$ induces an action of $\mathscr{G}(\bar{K} / \bar{F})$ on the columns of the matrix $B$ and thus the $p$-regular $\bar{F}$-classes of highest defect are in one-to-one correspondence with the orbits of columns relative to $\mathscr{G}(\bar{K} / \bar{F})$.

By a well-known permutation lemma of Brauer [1, Lemma 3], Theorem 7 is proven once we establish that $b_{i}^{\sigma} k\left(g_{j}\right)=b_{i}\left(g_{j}^{k}\right)$ for each $b_{i}\left(g_{j}\right)$ in the sum (4.1) and $\sigma_{k} \in \mathscr{C}\left(\bar{K} / \bar{F}^{i}\right)$. For non-p-regular $g, b_{i}(g)=0$. For $p$-regular $g$, we have $b_{i}(g)=\overline{c_{i}(g)}$ where $d_{i}=\sum_{g \in G} c_{i}(g) g$. But $d=$ $\Sigma \delta(\zeta)$ where $\delta(\zeta)$ is the primitive central idempotent in $E G$ with which $\zeta$ is associated and the sum is over all $\zeta$ belonging to $\bar{d}$. By $[2, \S 33]$

$$
\delta(\zeta)=\frac{\zeta(1)}{|G|} \sum_{g \in G} \zeta\left(g^{-1}\right) g .
$$

Thus

$$
c(g)=\sum_{\zeta \in \bar{d}} \frac{\zeta(1) \zeta\left(g^{-1}\right)}{|G|} .
$$

$K$ is unramified over $F$ and therefore $\mathscr{C}(K / F) \cong \mathscr{C}(\bar{K} / \bar{F})$ under the map $\sigma \rightarrow \bar{\sigma}$. It is easy to check that $\sigma_{k}$ is the element $\mathscr{G}(K / F)$ corresponding to the unit $k$ in $U_{m}$ if and only if $\bar{\sigma}_{k} \in \mathscr{C}(\bar{K} / \bar{F})$ corresponds to $k$. Thus

$$
\begin{aligned}
c_{i}^{\sigma k}(\delta) & =\sum_{\zeta \in \frac{1}{\omega_{i}}} \frac{\zeta(1) \zeta^{\sigma_{k}}\left(g^{-1}\right)}{|G|} \\
& =c_{i}\left(g^{k}\right)
\end{aligned}
$$

and therefore

$$
b_{i}^{\bar{\sigma} k}(g)=b_{i}\left(g^{k}\right)
$$

REMARK. If $L$ is any field of characteristic $p$, then $L=\bar{F}$ for some 
$F$ where $F$ is an unequal characteristic local field. [6, Theorem 2] Thus, Theorem 7 is actually a result on the number of blocks over an arbitrary field of characteristic $p$.

\section{REFERENCES}

1. Richard Brauer, On the connection between the ordinary and modular characters of groups of finite order, Ann. Math., 42 (1941), 926-935.

2. C. W. Curtis and I. Reiner, Representations Theory of Finite Groups and Associative Algebras, Interscience, New York, 1962.

3. Burton Fein, Representations of direct products of finite groups, Pacific J. Math., 20 (1967), 45-58.

4. Walter Feit, Characters of Finite Groups, W. A. Benjamin, New York, 1967.

5. J. A. Green, Blocks of modular representations, Math. Zeit., 79 (1962), 100-115.

6. S. MacLane, Subfields and automorphism groups of p-adic fields, Ann. Math., 40 (1939), 423-442.

7. W. F. Reynolds, Block idempotents and normal p-subgroups, Nagoya Math. J., 28 (1966), 1-13.

8. Blocks and F-class algebras of finite groups, Pacific J. Math., 38 (1971). 193-205.

9. A. Rosenberg, Blocks and centres of group algebras, Math. Zeit., 76 (1961), 209-216.

10. B. L. Van der Waerden. Modern Algebra, Vol. II, Ungar, New York, 1949.

11. E. Weiss, Algebraic Number Theory, McGraw-Hill, New York, 1963.

Received May 14, 1970. Research supported by the University of Delaware Research Foundation.

UnIVERsity of Delaware 



\section{PACIFIC JOURNAL OF MATHEMATICS}

\section{EDITORS}

H. SAMELSON

Stanford University

Stanford, California 94305

C. R. HOBBY

University of Washington

Seattle, Washington 98105
J. DugundJI

Department of Mathematics

University of Southern California

Los Angeles, California 90007

RICHARD ARENS

University of California

Los Angeles, California 90024

\section{ASSOCIATE EDITORS}

E. F. BeCKENBACH

B. H. NeumanN

F. WOLF

K. YosHIDA

\section{SUPPORTING INSTITUTIONS}

UNIVERSITY OF BRITISH COLUMBIA

CALIFORNIA INSTITUTE OF TECHNOLOGY

UNIVERSITY OF CALIFORNIA

MONTANA STATE UNIVERSITY

UNIVERSITY OF NEVADA

NEW MEXICO STATE UNIVERSITY

OREGON STATE UNIVERSITY

UNIVERSITY OF OREGON

OSARA UNIVERSITY
UNIVERSITY OF SOUTHERN CALIFORNIA STANFORD UNIVERSITY

UNIVERSITY OF TOKYO

UNIVERSITY OF UTAH

WASHINGTON STATE UNIVERSITY

UNIVERSITY OF WASHINGTON

AMERICAN MATHEMATICAL SOCIETY

NAVAL WEAPONS CENTER

Printed in Japan by International Academic Printing Co., Ltd., Tokyo, Japan 


\section{Pacific Journal of Mathematics}

\section{Vol. 40, No. $1 \quad$ September, 1972}

Alex Bacopoulos and Athanassios G. Kartsatos, On polynomials

approximating the solutions of nonlinear differential equations........

Monte Boisen and Max Dean Larsen, Prüfer and valuation rings with zero

divisors ..........................................

James J. Bowe, Neat homomorphisms

David W. Boyd and Hershy Kisilevsky, The Diophantine equation

$$
u(u+1)(u+2)(u+3)=v(v+1)(v+2) \ldots \ldots \ldots \ldots \ldots \ldots \ldots
$$

George Ulrich Brauer, Summability and Fourier analysis ...............

Robin B. S. Brooks, On removing coincidences of two maps when only one,

rather than both, of them may be deformed by a homotopy ............

Frank Castagna and Geert Caleb Ernst Prins, Every generalized Petersen

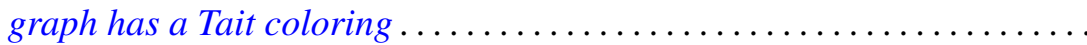

Micheal Neal Dyer, Rational homology and Whitehead products ..........

John Fuelberth and Mark Lawrence Teply, The singular submodule of a

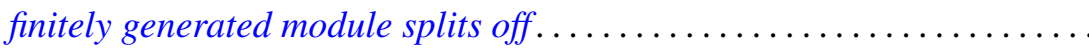

Robert Gold, $\Gamma$-extensions of imaginary quadratic fields ............ 83

Myron Goldberg and John W. Moon, Cycles in k-strong tournaments.......

Darald Joe Hartfiel and J. W. Spellmann, Diagonal similarity of irreducible

matrices to row stochastic matrices...............

Wayland M. Hubbart, Some results on blocks over local fields ..

Alan Loeb Kostinsky, Projective lattices and bounded homomorphisms....

Kenneth O. Leland, Maximum modulus theorems for algebras of operator

valued functions ...

Jerome Irving Malitz and William Nelson Reinhardt, Maximal models in the

language with quantifier "there exist uncountably many" ..

John Douglas Moore, Isometric immersions of space forms in space

forms.

Ronald C. Mullin and Ralph Gordon Stanton, A map-theoretic approach to

Davenport-Schinzel sequences ....................

Chull Park, On Fredholm transformations in Yeh-Wiener space. .

Stanley Poreda, Complex Chebyshev alterations ..............

Ray C. Shiflett, Extreme Markov operators and the orbits of Ryff. ...

Robert L. Snider, Lattices of radicals .....................

Ralph Richard Summerhill, Unknotting cones in the topological

category ................................

Charles Irvin Vinsonhaler, A note on two generalizations of $\mathrm{QF}-3 \ldots \ldots 229$

William Patterson Wardlaw, Defining relations for certain integrally

parameterized Chevalley groups...................

William Jennings Wickless, Abelian groups which admit only nilpotent

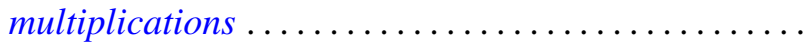

